\title{
Caracterização morfológica, produtividade e rendimento comercial de cultivares de alho
}

\author{
Juliano Tadeu V de Resende'; Rafael Gustavo F Morales'; Daniel S Zanin ${ }^{1}$; Francisco V Resende ${ }^{2}$; \\ Juliana T de Paula ${ }^{1}$; Diego M Dias ${ }^{1}$; Alexandre G Galvão ${ }^{1}$ \\ 'UNICENTRO-Dep ${ }^{\text {to }}$ Agronomia, Campus Univ. CEDETEG, C. Postal 3010, 85040-080 Guarapuava-PR; jresende@unicentro.br; \\ moralescefet@yahoo.com.br; zanin.suek@hotmail.com; juliana.tauffer@hotmail.com; diegomunhozdias@hotmail.com; galvaox@gmail. \\ com; ${ }^{2}$ Embrapa Hortaliças, C. Postal 218, 70351-970 Brasília-DF; fresende@cnph.embrapa.br
}

\begin{abstract}
RESUMO
O Brasil tem condições de elevar a produção de alho a ponto de suprir a demanda interna. Para alcançar tal êxito, é necessário avaliar o maior número possível de cultivares visando estabelecer as melhores para cada região. Sendo assim, esse trabalho objetivou avaliar o rendimento econômico e a qualidade de bulbos de 20 cultivares de alho na região Centro-Sul do Paraná. O experimento foi conduzido entre os meses de março e setembro de 2009, no município de Guarapuava-PR. O delineamento experimental foi em blocos casualizados, com três repetições. Os tratamentos constituíram-se em doze cultivares de alho seminobre e oito cultivares de alho nobre. Foram realizadas as avaliações de produtividade total (PT), produção comercial (PC) e classificação comercial dos bulbos; superbrotamento; e seis caracteres relacionados à morfologia da planta: diâmetro do pseudocaule, comprimento e largura da folha, comprimento, largura e número de bulbilhos. As cultivares do grupo nobre apresentaram PT superior às cultivares do grupo seminobre, porém, não houve diferença com relação à PC. Entre as cultivares do grupo nobre, a São Valentim apresentou a maior PC de bulbos, com $8,9 \mathrm{t} \mathrm{ha}^{-1}$. As cultivares do grupo nobre apresentaram maior porcentagem de bulbos nas classes 6 e 7, com destaque para as cultivares Chonan, com 40,3\% dos bulbos na classe 7 e a cultivar Ito, com $75 \%$ dos bulbos nas classes 6 e $7 . \mathrm{O}$ oposto foi observado no grupo seminobre, pois a maioria dos bulbos encontra-se nas classes 3, 4 e 5. Dentre as cultivares avaliadas, a cultivar São Valentim se adaptou melhor às condições edafoclimáticas da região, apresentando elevado potencial produtivo.
\end{abstract}

Palavras-chave: Allium sativum, superbrotamento, alho nobre, alho seminobre.

\begin{abstract}
Morphological characterization, commercial yield and productivity of garlic cultivars

Brazil could increase garlic production up to the point of supplying the internal demand. To achieve this success, it is necessary to evaluate the maximum number of cultivars to establish the best ones for each region. Therefore, we aimed to evaluate economic yield and quality of bulbs of 20 garlic cultivars in Center-South Paraná state, Brazil. The experiment was carried out between March and September 2009, in Guarapuava, Paraná state. Experimental design was randomized blocks with three replications. The treatments consisted of twelve seminoble and eight noble garlic cultivars. We evaluated the total production (TP), commercial production (CP) and commercial classification of bulbs; secondary bulb growth; and six characteristics related to the plant morphology: pseudostem diameter, length and leaf width, length, width and number of cloves. Noble group cultivars have presented TP superior to seminoble cultivars. However, there was no difference in relation to CP. Among cultivars of noble group, San Valentim presented greater CP bulbs, with 8,9 $\mathrm{t}$ $\mathrm{ha}^{-1}$. Noble group cultivars have presented higher percentual of bulbs in classes 6 and 7, especially the cultivars Chonan, with $40.3 \%$ of the bulbs in the class 7 and cultivar Ito, with $75 \%$ of the bulbs in classes 6 and 7. The opposite was observed in the seminoble group, since most bulbs of this group were found in classes 3, 4 and 5 . Among the evaluated cultivars, San Valentin showed to be better adapted to the edafoclimatic conditions of the region, presenting a great yielding potential.
\end{abstract}

Keywords: Allium sativum, secondary bulb growth, noble garlic, seminoble garlic.

(Recebido para publicação em 14 de setembro de 2011; aceito em 18 de dezembro de 2012)

(Received on September 14, 2011; accepted on December 18, 2012)

$\mathrm{O}$ Brasil é o segundo maior consumidor e o maior importador de alho (Allium sativum) do mundo (Souza \& Macêdo, 2009). Apesar da crescente oferta do produto no mercado interno, a produção brasileira é insuficiente para atender à demanda nacional (Backes et al., 2008), devido à baixa produtividade $\left(8,2 \mathrm{t} \mathrm{ha}^{-1}\right)$ (Souza \& Macêdo, 2009). O Brasil tem condições de elevar sua produção a ponto de suprir a demanda interna e até mesmo tornar-se exporta- dor do produto. Uma das vertentes para alcançar tal êxito é a avaliação do maior número possível de cultivares visando estabelecer as melhores para cada região e época de cultivo. Entretanto, não basta o rendimento quantitativo, pois são necessárias pesquisas que identifiquem as cultivares e técnicas que produzam bulbos com baixa incidência de defeitos (Trevisan et al., 1997; Macedo et al., 2006).

Existem no Brasil diversas cultivares disponíveis ao produtor, que variam em produção, morfologia e fisiologia da planta e do bulbo. Essas cultivares são divididas em dois grandes grupos: nobres e seminobres. As cultivares do grupo nobre são caracterizadas por possuírem bulbos redondos uniformes, bulbilhos grandes com ausência de palitos e sensibilidade ao superbrotamento. Os bulbos têm túnica branca e película de cor rósea ou roxa e os bulbilhos têm película rósea escura, necessitando de 
vernalização para plantio nas regiões do Cerrado Brasileiro (Mota et al., 2004, 2006). O alho seminobre possui bulbos com formato irregular, maior número de bulbilhos, presença de palitos, túnica branca com película branca a levemente arroxeada, não necessita de vernalização para produzir bulbos em regiões tropicais (Mota et al., 2004, 2006). Além disso, algumas cultivares desse grupo (Amarante, Gravatá, Gigante Roxo, Gigante do Núcleo e Gigante Curitibanos) apresentam bulbos ovalados com cerca de 12 bulbilhos graúdos e resistência ao superbrotamento, podendo ser boa opção para pequeno produtor e cultivo orgânico.

O superbrotamento é um dos principais fatores responsáveis pela redução da produtividade, podendo servir como indicativo da baixa adaptabilidade de uma cultivar a uma região. Vários fatores podem estar relacionados ao superbrotamento, tais como fotoperíodo, temperatura, cultivar, adubação nitrogenada e irrigação (Macêdo et al., 2006). O manejo da irrigação, por meio de um déficit hídrico de 8 a 20 dias no período de bulbificação, reduz a taxa de superbrotamento e aumenta a produção comercial de bulbos (Souza \& Macedo, 2009). Por outro lado, o excesso de umidade aumenta o superbrotamento e compromete a produção comercial. De fato, talvez esse seja o maior problema encontrado pelos alhicultores da região Centro-Sul do estado do Paraná, em que o período de bulbificação e colheita frequentemente coincidem com o período chuvoso.

A avaliação de cultivares quanto à produtividade e qualidade de bulbos deve merecer destaque nas regiões produtoras ou que vislumbrem a possibilidade em ter o cultivo de alho como atividade econômica (Feitosa et al., 2009). Avaliando-se cinco cultivares de alho na região paulista de Tietê, observaram-se produtividades variando entre 5,84 a 8,44 $\mathrm{t} \mathrm{ha}^{-1}$, sendo a cultivar Jonas a mais indicada para cultivo na região (Trani et al., 2008). Comparando-se sete cultivares de alho em Lavras-MG, observaram-se produtividades variando entre 7,37 e 14,72 $\mathrm{t} \mathrm{ha}^{-1}$, concluindo que as cultivares Gigante Curitibanos e Gigante Roxo eram as mais promissoras para cultivo nas condições do Sul de Minas Gerais (Resende et al., 2003). Avaliando-se duas cultivares de alho em função da época de plantio e do período de vernalização em Guarapuava, observou-se produtividade variando de 8 a 13 t ha $^{-1}$, concluindo que o plantio no mês de março e 40 dias de vernalização são favoráveis para produção de bulbos com maior padrão comercial (Resende et al., 2011). Contudo, esses valores de produtividade não refletem a realidade do estado do Paraná, que possui produtividade média de 2,99 $\mathrm{t} \mathrm{ha}^{-1}$ (CONAB, 2011). Essa baixa produtividade se deve à falta de pesquisas na região, vinculado ao baixo uso de tecnologias e o desconhecimento por parte de produtores e extensionistas das melhores cultivares a serem cultivadas nas condições de solo e clima da região.

O objetivo do presente trabalho foi avaliar 20 cultivares de alho, sendo 12 seminobres e 8 nobres, quanto ao rendimento agronômico, qualidade de bulbos e aspectos morfológicos quando cultivadas no Centro-Sul do estado do Paraná.

\section{MATERIAL E MÉTODOS}

O experimento foi conduzido no município de Guarapuava em campo da Universidade Estadual do Centro-Oeste (UNICENTRO), Paraná (25'23'36'S, $51^{\circ} 27^{\prime} 19^{\prime \prime} \mathrm{O}$, altitude $\left.1.025 \mathrm{~m}\right)$. O clima da região é classificado como $\mathrm{Cfb}$ (Subtropical mesotérmico úmido), sem estação seca, com verões frescos e inverno moderado, conforme a classificação de Köppen. O solo é classificado como Latossolo Bruno Distroférrico típico, com textura muito argilosa (Embrapa, 2006). A precipitação durante a execução do experimento foi de $1202 \mathrm{~mm}$, temperatura média mínima de $13,5^{\circ} \mathrm{C}$, temperatura média máxima de $24,6^{\circ} \mathrm{C}$ e umidade relativa do ar de $81,9 \%$.

$\mathrm{O}$ delineamento experimental foi em blocos casualizados, com três repetições. Os tratamentos constituíram-se em doze cultivares de alho seminobre (Amarante, Gigante Lavínia, Gigante Roxão, Gravatá, Chinês Real, Chinês São Joaquim, Hozan, Caturra, Cateto Roxo, Gigante Roxo, Peruano e Gigante do Núcleo) e oito cultivares de alho nobre (Chonan, Roxo Pérola de Caçador, Quitéria, Jonas, Ito, São Valentim, Bergamota e Roxo Caxiense), todas dispostas na mesma área experimental constituindo um único experimento.

O plantio foi realizado em 23 de março de 2009, na profundidade de 5 $\mathrm{cm}$, com espaçamento de $20 \mathrm{~cm}$ entre linhas e $10 \mathrm{~cm}$ entre plantas, em parcelas de $2,0 \mathrm{~m}^{2}$, totalizando 100 plantas por parcela. A adubação de plantio foi realizada com base na análise do solo, utilizando-se, por parcela, $200 \mathrm{~g}$ do formulado NPK 4-14-8. A adubação de cobertura foi realizada aos 20, 40 e 60 dias após o plantio, com $12 \mathrm{~g}$ de uréia e 16 $\mathrm{g}$ de cloreto de potássio por parcela. $\mathrm{O}$ controle fitossanitário foi realizado com três pulverizações de Mancozeb $(2,0 \mathrm{~kg}$ $\left.\mathrm{ha}^{-1}\right)$, visando prevenir o aparecimento de ferrugem (Puccinia allii) e alternária (Alternaria porri). Para o controle de ácaros e tripes foi aplicado Thiometon a cada 30 dias. Antes do plantio os bulbilhos foram tratados com solução de 2,5\% de Iprodione, para prevenir possível ataque de patógenos de solo. A cultura foi mantida livre de plantas daninhas por meio de capinas manuais.

O ponto de colheita foi determinado pelo amarelecimento e secamento parcial da parte aérea e/ou tombamento das plantas. Esse estádio foi observado entre os dias 15 e 30 de agosto e 01 e 20 de setembro para as cultivares do grupo seminobre e nobre, respectivamente. Após a colheita, as plantas foram submetidas ao processo de cura por 40 dias, sendo então determinada a produtividade total em $\mathrm{t} \mathrm{ha}^{-1} \mathrm{e}$ a porcentagem de bulbos superbrotados, pela contagem dos bulbos com três ou mais folhas superbrotadas após a cura, expressando os dados em relação ao número total de bulbos colhidos. Os bulbos adequados à comercialização foram distribuídos em classes de tamanho conforme o diâmetro transversal: 7 (>56 mm), 6 (47-56 mm), 5 (42-47 mm), 4 (37-42 mm) e 3 (32-37 $\mathrm{mm}$ ), segundo a portaria $\mathrm{n}^{\mathrm{o}} 242$, de 17 de setembro de 1992, do Ministério da Agricultura, Pecuária e Abastecimento (MAPA) (Menezes Sobrinho, 1997).

Foram avaliados sete caracteres morfológicos relacionados à planta, folha e bulbilho. Aos 100 dias após o plantio mediu-se o comprimento da maior folha 
e a sua largura na porção mediana de dez plantas ao acaso. Nessas mesmas plantas, com um paquímetro digital de duas casas decimais de precisão, mediu-se o diâmetro do pseudocaule, aproximadamente a $5 \mathrm{~cm}$ do colo da planta. Após a cura, dez bulbos amostrados na parcela foram debulhados para a medição do comprimento e largura dos bulbilhos, com o auxílio do paquímetro digital, contando-se após a medição o número total de bulbilhos por bulbo.

Os dados foram submetidos à análise de variância e as médias comparadas pelo teste de Scott-Knott com 5\% de probabilidade. As cultivares foram agrupadas nos grupos nobre e seminobre, sendo as médias dos grupos comparadas pelo teste $t(p \leq 0,05)$.

\section{RESULTADOS E DISCUSSÃO}

As cultivares do grupo nobre apresentaram em média maior produtividade total (PT) $\left(5,4\right.$ t ha $\left.^{-1}\right)$ do que as cultivares do grupo seminobre (3,5 tha-1), contudo, não houve diferença entre as cultivares dos dois grupos com relação à produção comercial (PC) $\left(2,8\right.$ e $2,4 \mathrm{t} \mathrm{ha}^{-1}$, respectivamente) (Tabela 1). No grupo nobre, a cultivar São Valentim apresentou a maior PT (10,6 tha-1) e PC $\left(8,9 \mathrm{t} \mathrm{ha}^{-1}\right) \mathrm{de}$ bulbos, demonstrando elevado potencial para cultivo na região. Esses valores são superiores a média produtiva do estado do Paraná para o ano de 2011 (2,99 t $\mathrm{ha}^{-1}$ ), e inferiores aos observados por Resende et al. (2011) com as cultivares Roxo Pérola de Caçador e Quitéria na mesma região, que obtiveram $\mathrm{PC}$ variando de 8 a $13 \mathrm{t} \mathrm{ha}^{-1}$. Por outro lado, as cultivares Bergamota e Roxo Caxiense apresentaram as menores produtividades, indicando que essas cultivares não se adaptaram ao clima da região. Dentro do grupo seminobre, a cultivar Gravatá se destacou com PT de 5,9 t ha-1, produtividade semelhante à maioria das cultivares do grupo nobre e um pouco superior a média do estado. Apesar de apresentar maior PT, essa cultivar não diferiu na $\mathrm{PC}$ em relação às cultivares Caturra, Chinês São Joaquim e Cateto Roxo. As PC das cultivares do grupo seminobre foram baixas, principalmente das cultivares Peruano, Hozan e Chinês Real.

Tabela 1. Características agronômicas de cultivares de alho cultivadas na região Centro-Sul do Paraná (agronomic characteristics of garlic cultivars cultivated in Paraná Center South region). Guarapuava, UNICENTRO, 2009.

\begin{tabular}{|c|c|c|c|}
\hline \multirow{2}{*}{ Cultivares } & \multicolumn{2}{|c|}{ Produtividade } & \multirow{2}{*}{$\begin{array}{c}\text { Super } \\
\text { brotamento }(\%)\end{array}$} \\
\hline & total $\left(\mathrm{t} \mathrm{ha}^{-1}\right)$ & comercial $\left(\mathrm{t} \mathrm{ha}^{-1}\right)$ & \\
\hline \multicolumn{4}{|l|}{ Grupo nobre } \\
\hline Caçador & $5,8 \mathrm{~b}$ & $2,4 \mathrm{c}$ & $61,8 \mathrm{~b}$ \\
\hline São Valentim & $10,6 \mathrm{a}$ & $8,9 \mathrm{a}$ & $16,2 \mathrm{c}$ \\
\hline Bergamota & $2,4 \mathrm{~d}$ & $0,3 \mathrm{~d}$ & $99,2 \mathrm{a}$ \\
\hline Ito & $6,4 \mathrm{~b}$ & $2,0 \mathrm{c}$ & $71,6 \mathrm{a}$ \\
\hline Jonas & $6,1 \mathrm{~b}$ & $2,6 \mathrm{c}$ & $56,6 \mathrm{~b}$ \\
\hline Roxo Caxiense & $2,1 \mathrm{~d}$ & $0,3 \mathrm{~d}$ & $97,5 \mathrm{a}$ \\
\hline Quitéria & $3,3 \mathrm{c}$ & $1,7 \mathrm{c}$ & $26,6 \mathrm{~b}$ \\
\hline Chonan & $6,7 \mathrm{~b}$ & $3,7 \mathrm{~b}$ & $34,7 \mathrm{~b}$ \\
\hline Média & $5,4 \mathrm{~A}$ & $2,8 \mathrm{~A}$ & $58,0 \mathrm{~A}$ \\
\hline \multicolumn{4}{|l|}{ Grupo seminobre } \\
\hline Chinês Real & $4,2 \mathrm{c}$ & $0,7 \mathrm{~d}$ & $76,7 \mathrm{a}$ \\
\hline Amarante & $2,5 \mathrm{~d}$ & $2,2 \mathrm{c}$ & $19,8 \mathrm{c}$ \\
\hline Hozan & $3,1 \mathrm{~d}$ & $0,7 \mathrm{~d}$ & $73,2 \mathrm{a}$ \\
\hline Caturra & $4,2 \mathrm{c}$ & $4,2 \mathrm{~b}$ & $0,0 \mathrm{c}$ \\
\hline Gravatá & $5,9 \mathrm{~b}$ & $5,2 \mathrm{~b}$ & $9,2 \mathrm{c}$ \\
\hline $\begin{array}{l}\text { Gigante do Nú- } \\
\text { cleo }\end{array}$ & $4,1 \mathrm{c}$ & $1,7 \mathrm{c}$ & $61,6 \mathrm{~b}$ \\
\hline Chinês S.Joaquim & $4,7 \mathrm{c}$ & $3,7 \mathrm{~b}$ & $12,0 \mathrm{c}$ \\
\hline Cateto Roxo & $4,4 \mathrm{c}$ & $3,9 \mathrm{~b}$ & $5,3 \mathrm{c}$ \\
\hline Gigante Lavínia & $3,7 \mathrm{c}$ & $2,4 \mathrm{c}$ & $23,1 \mathrm{~b}$ \\
\hline Peruano & $1,7 \mathrm{~d}$ & $0,2 \mathrm{~d}$ & 87,1 a \\
\hline Gigante Roxo & $1,3 \mathrm{~d}$ & $1,1 \mathrm{~d}$ & $14,4 \mathrm{c}$ \\
\hline Gigante Roxão & $2,7 \mathrm{~d}$ & $2,7 \mathrm{c}$ & $0,0 \mathrm{c}$ \\
\hline Média & $3,5 \mathrm{~B}$ & $2,4 \mathrm{~A}$ & $31,9 \mathrm{~B}$ \\
\hline
\end{tabular}

${ }^{1}$ Médias seguidas pela mesma letra minúscula na coluna não diferem entre si pelo teste de Scott-Knott $(\mathrm{p}<0,05)$; ${ }^{2}$ Médias dos grupos nobre e seminobre acompanhadas pela mesma letra maiúscula, na coluna, não diferem entre si pelo teste $t(p \leq 0,05)$ ['average values followed by the same lowercase letter did not differ among themselves by the Scott-Knott test $(p<0,05) ;{ }^{2}$ Average values of noble and seminoble garlic group accompanied from the same capital letter, in the column, do not differ among themselves by the test $t(p \leq 0,05)]$.

As baixas produtividades podem estar relacionadas à sensibilidade que as cultivares de alho têm aos fatores climáticos, como fotoperíodo e temperatura (Souza \& Macêdo, 2009), tornando uma cultivar produtiva em algumas regiões e improdutivas em outras. $\mathrm{O}$ fotoperíodo durante a bulbificação foi de aproximadamente $9 \mathrm{~h} 40 \mathrm{~min}$, inferior ao fotoperíodo crítico das cultivares nobres, que é de 13 horas de luz, justificando nesse caso a baixa produtividade desse grupo (Souza \& Macêdo, 2009). O fotoperíodo consiste em um importante fator para que se possa estabelecer a melhor época de plantio e cultivar para cada região, pois além de causar o estímulo para bulbificação, está relacionado diretamente com o acúmulo de fotoassimilados mediante processo fotossintético (Lacher, 2000). No presente trabalho, observou-se que o fotoperíodo da região na época de cultivo seria inadequado para cultivares do grupo nobre, o que poderia ocasionar um crescimento vegetativo, sem que houvesse a formação normal de bulbos. No entanto, apesar do fotoperíodo inadequado, foi observada uma produtividade acima da média do estado do Paraná, o que pode ser explicado pelo fato das baixas temperaturas observadas promoverem interação com 
Tabela 2. Classificação comercial de cultivares de alho cultivadas na região Centro-Sul do Paraná (commercial classification of garlic cultivars in Paraná Center South region). Guarapuava-PR, UNICENTRO, 2009.

\begin{tabular}{|c|c|c|c|c|c|}
\hline \multirow{2}{*}{ Cultivares } & \multicolumn{5}{|c|}{ Classificação comercial (\%) } \\
\hline & 3 & 4 & 5 & 6 & 7 \\
\hline \multicolumn{6}{|l|}{ Grupo nobre } \\
\hline Caçador & $14,0 \mathrm{bC}$ & $23,0 \mathrm{bA}$ & $18,4 \mathrm{aB}$ & $24,7 \mathrm{aA}$ & $20,0 \mathrm{cB}$ \\
\hline São Valentim & $13,6 \mathrm{bC}$ & $27,7 \mathrm{aA}$ & $26,7 \mathrm{aA}$ & $21,8 \mathrm{aB}$ & $10,3 \mathrm{cC}$ \\
\hline Bergamota & $7,8 \mathrm{bD}$ & $4,2 \mathrm{bD}$ & $14,0 \mathrm{aC}$ & $31,7 \mathrm{aB}$ & $42,3 \mathrm{bA}$ \\
\hline Ito & $2,8 \mathrm{bC}$ & $8,6 \mathrm{bC}$ & $14,2 \mathrm{aC}$ & $19,2 \mathrm{aB}$ & $55,2 \mathrm{aA}$ \\
\hline Jonas & $8,6 \mathrm{bC}$ & $19,6 \mathrm{bB}$ & $14,3 \mathrm{aC}$ & $21,3 \mathrm{aB}$ & $36,1 \mathrm{bA}$ \\
\hline Roxo Caxiense & $0,8 \mathrm{bC}$ & $14,9 \mathrm{bC}$ & $8,5 \mathrm{aC}$ & $21,7 \mathrm{aB}$ & $54,1 \mathrm{aA}$ \\
\hline Quitéria & $22,3 \mathrm{aA}$ & $22,1 \mathrm{bA}$ & $19,1 \mathrm{aA}$ & $15,7 \mathrm{aB}$ & $20,8 \mathrm{cA}$ \\
\hline Chonan & $6,7 \mathrm{bC}$ & $10,1 \mathrm{bC}$ & $20,4 \mathrm{aB}$ & $22,5 \mathrm{aB}$ & $40,3 \mathrm{bA}$ \\
\hline Média & $9,6 \mathrm{D}$ & $16,29 \mathrm{C}$ & $16,9 \mathrm{C}$ & $22,3 \mathrm{~B}$ & $34,9 \mathrm{~A}$ \\
\hline \multicolumn{6}{|l|}{ Grupo seminobre } \\
\hline Chinês Real & $27,2 \mathrm{aA}$ & $29,7 \mathrm{aA}$ & $22,8 \mathrm{aA}$ & $16,6 \mathrm{aB}$ & $3,7 \mathrm{dC}$ \\
\hline Amarante & $30,1 \mathrm{aB}$ & $38,2 \mathrm{aA}$ & $24,0 \mathrm{aB}$ & $7,8 \mathrm{bC}$ & $0,0 \mathrm{dD}$ \\
\hline Hozan & $27,2 \mathrm{aA}$ & $33,0 \mathrm{aA}$ & $28,0 \mathrm{aA}$ & $10,6 \mathrm{bB}$ & $1,2 \mathrm{dC}$ \\
\hline Caturra & $17,0 \mathrm{bC}$ & $24,0 \mathrm{bB}$ & 29,4 aA & $22,7 \mathrm{aB}$ & $7,0 \mathrm{dD}$ \\
\hline Gravatá & 34,9 aA & $39,4 \mathrm{aA}$ & $14,3 \mathrm{aB}$ & $11,5 \mathrm{bB}$ & $0,0 \mathrm{dC}$ \\
\hline Gigante do Núcleo & $15,2 \mathrm{bB}$ & $24,6 \mathrm{bA}$ & $21,0 \mathrm{aA}$ & $22,9 \mathrm{aA}$ & $16,4 \mathrm{cB}$ \\
\hline Chinês São Joaquim & $21,7 \mathrm{aB}$ & $41,0 \mathrm{aA}$ & $28,4 \mathrm{aB}$ & $8,1 \mathrm{bC}$ & $0,9 \mathrm{dC}$ \\
\hline Cateto Roxo & $41,6 \mathrm{aA}$ & $34,2 \mathrm{aB}$ & $18,2 \mathrm{aC}$ & $3,6 \mathrm{bD}$ & $2,4 \mathrm{dD}$ \\
\hline Gigante Lavínia & $17,5 \mathrm{bB}$ & $31,0 \mathrm{aA}$ & $31,8 \mathrm{aA}$ & $14,8 \mathrm{bB}$ & $4,9 \mathrm{dC}$ \\
\hline Peruano & $38,7 \mathrm{aA}$ & $24,2 \mathrm{bB}$ & $16,1 \mathrm{aC}$ & $21,0 \mathrm{aB}$ & $0,0 \mathrm{dD}$ \\
\hline Gigante Roxo & $25,7 \mathrm{aC}$ & $36,4 \mathrm{aA}$ & $30,3 \mathrm{aA}$ & $7,6 \mathrm{bD}$ & $0,0 \mathrm{dE}$ \\
\hline Gigante Roxão & $24,3 \mathrm{aB}$ & $44,5 \mathrm{aA}$ & $25,6 \mathrm{aB}$ & $5,5 \mathrm{bC}$ & $0,0 \mathrm{dD}$ \\
\hline Média & $26,7 \mathrm{~B}$ & $33,3 \mathrm{~A}$ & $24,2 \mathrm{~B}$ & $12,7 \mathrm{C}$ & $3,0 \mathrm{D}$ \\
\hline
\end{tabular}

Médias seguidas pela mesma letra maiúscula na linha e minúscula na coluna não diferem entre si pelo teste de Scott-Knott $(\mathrm{p} \leq 0,05)$ [average values followed by the same capital letter in the line and lowercase in the column, do not differ among themselves by the ScottKnott test $(\mathrm{p} \leq 0,05)]$.

o fotoperíodo, de forma compensatória (Souza \& Macêdo, 2009), estimulando a diferenciação das gemas axilares e o início da bulbificação, proporcionando a formação dos bulbos, mesmo sob condições de luminosidade insuficientes.

Nas cultivares seminobres observou-se uma condição contrária ao verificado para o grupo dos alhos nobres. Apesar do fotoperíodo adequado na região para as cultivares seminobres, que apresentam fotoperíodo crítico superior a 9 horas, ressalta-se que as baixas temperaturas durante o ciclo da cultura contribuíram para que esse grupo tivesse um aumento no ciclo de cultivo e no número de bulbilhos por bulbo, resultando em menor superbrotamento, que foi de $99,2 \%$ e $97,5 \%$, respectivamente. O mesmo pode ser observado com as cultivares Peruano, Chinês Real e Hozan, as menos produtivas do grupo seminobre e com superbrotamento de $87,1 \%, 76,7 \%$ e $73,2 \%$, respectivamente. Essa relação entre queda da produção comercial devido ao aumento do superbrotamento foi relatada por outros autores que observaram correlação entre o aumento do superbrotamento e a redução da PC dos bulbos (Resende \& Souza, 2001; Resende et al., 2011). Outros fatores também podem afetar a PC e causar anomalias nos bulbos, como, por exemplo, a não adaptação das cultivares às condições edafoclimáticas do local de cultivo (Souza \& Macêdo, 2009), solo muito pesado ou compactado (Filgueira, 2008), excesso de chuvas ou de irrigação (Macêdo et al., 2006), cultivar e período de vernalização dos bulbos (Souza \& Macêdo, 2004). Neste estudo, a pluviosidade acima da média (aproximadamente $50 \mathrm{~mm} / \mathrm{mês}$ ) durante o final do ciclo, aliada aos fatores fotoperíodo e temperatura inadequadas durante alguns momentos do ciclo, foram responsáveis por ter influenciado na elevada taxa de superbrotamento dos bulbos. A insuficiência do fotoperíodo para as cultivares de alho nobre durante o ciclo, pode ter sido um dos fatores que mais contribuiu para o aumento da taxa de superbrotamento, pois ocorre supressão desta anomalia quando o cultivo ocorre em fotoperíodo acima do crítico (Souza \& Macêdo, 2009). Baixas temperaturas ao longo do ciclo também contribuíram para aumento do superbrotamento (Carmo, 1984), explicando assim o alto índice de superbrotamento detectado para algumas cultivares desse grupo, conforme citado anteriormente.

A diferença entre as cultivares nobres e seminobres fica evidente pela classificação comercial dos bulbos (Tabela 2). Com base nas médias de todas as cultivares para classe comercial, as pertencentes ao grupo nobre apresentaram maior porcentagem de bulbos nas classes 6 e 7, classes de maior relevância para o mercado, visto que os maiores bulbos recebem as melhores cotações pelo mercado consumidor (Resende et al., 2003). O oposto é observado no 
Tabela 3. Resultados da avaliação agronômica de características vegetativas e reprodutivas de cultivares de alho (results of the agronomic evaluation os vegetative and reproductive characteristics of garlic cultivars). Guarapuava, UNICENTRO, 2009.

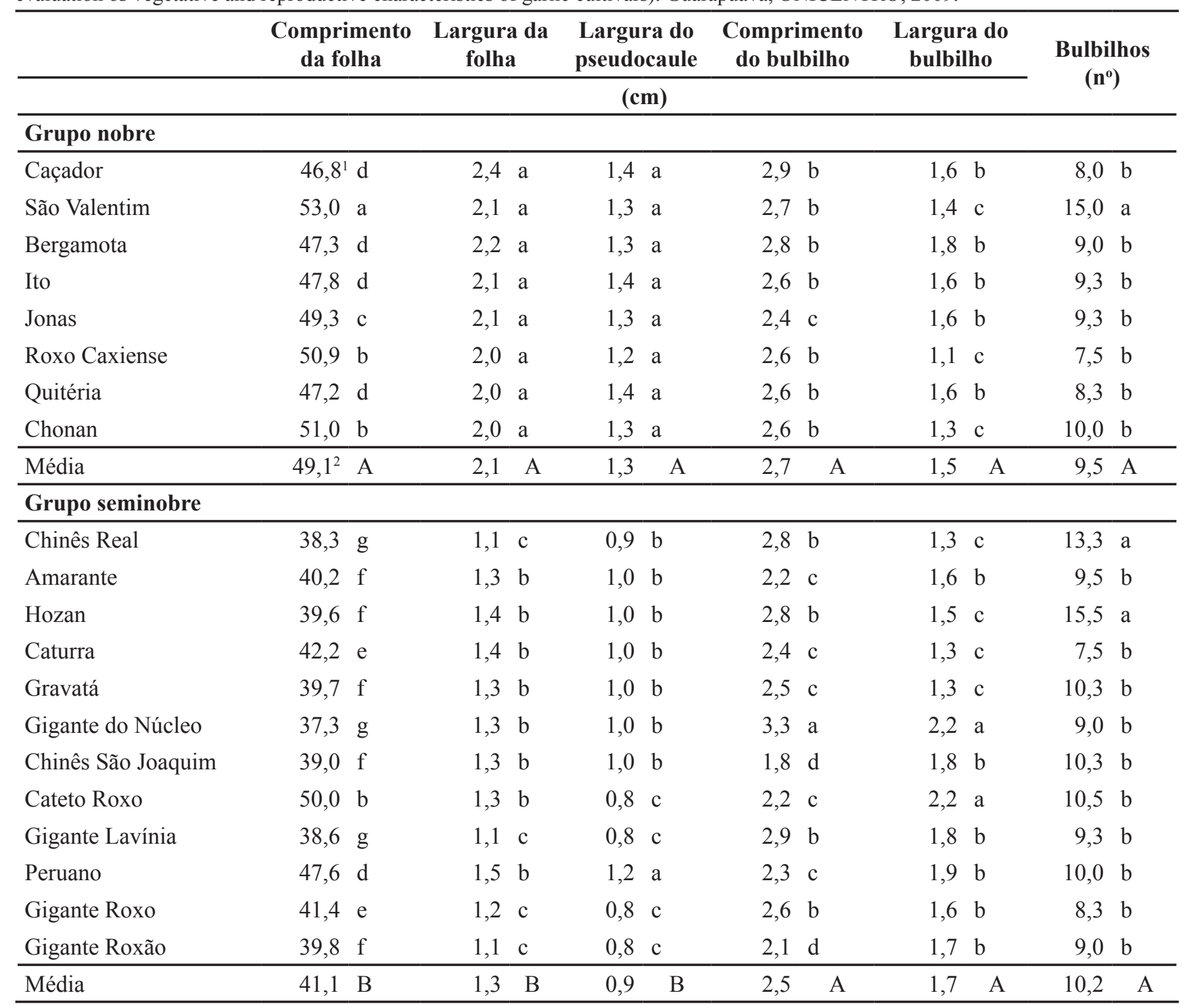

${ }^{1}$ Médias seguidas pela mesma letra minúscula na coluna não diferem estatisticamente pelo teste de Skott-Knott ( $\left.\mathrm{p} \leq 0,05\right) ;{ }^{2}$ Médias dos grupos nobre e seminobre acompanhadas pela mesma letra maiúscula, na coluna, não diferem entre si pelo teste $t(p \leq 0,05)\left[{ }^{1}\right.$ average values followed by same lowercase letters in column do not differ statistically according to Skott-Knott test ( $\mathrm{p} \leq 0,05) ;{ }^{2}$ Average values of noble and seminoble group accompanied from the same capital letter, in the column, do not differ among themselves by the test $t(p \leq 0,05)]$.

grupo seminobre, pois a maioria dos bulbos encontra-se nas classes 3, 4 e 5 (Tabela 2). Resultados semelhantes foram observados por outros autores, que avaliaram três cultivares de alho seminobre e observaram maior porcentagem de bulbos na classe 4 (Trani et al., 2008). A cultivar Chonan, com $40,3 \%$ dos bulbos na classe 7, e a cultivar Ito, com $75 \%$ dos bulbos nas classes $6 \mathrm{e}$ 7 , se destacaram entre as cultivares nobres pela qualidade dos bulbos. São Valentim, que foi a mais produtiva, apresenta seus bulbos distribuídos nas cinco classes, com pequenas diferenças entre classes. As cultivares mais produtivas do grupo seminobre, Gravatá, Chinês São Joaquim, Caturra e Cateto Roxo apresentaram a maioria dos seus bulbos nas classes 3, 4 e 5 .

A superioridade das cultivares do grupo nobre na PT de bulbos em relação às cultivares seminobres pode estar relacionada à morfologia da planta. As cultivares do grupo nobre apresentaram folhas mais largas que cultivares do grupo seminobre, com média de 2,1 $\mathrm{cm}$ e $1,3 \mathrm{~cm}$, respectivamente (Tabela
3). Esses valores foram inferiores aos observados em outros trabalhos com as cultivares seminobres Gigante Curitibanos e Gigante Roxo, em que a largura da folha foi de 2,7 e 3,1 cm, respectivamente (Mota et al., 2005). A largura da folha da cultivar Gigante Roxo nesse estudo foi de $1,2 \mathrm{~cm}$, menos da metade do observado pelo autor supracitado. Essa diferença pode ser atribuída à plasticidade morfológica das cultivares de alho que respondem diferentemente em cada região de cultivo. $\mathrm{O}$ mesmo pode ser observado para o diâmetro do 
pseudocaule e comprimento da folha, em que as cultivares do grupo nobre apresentaram valores superiores aos observados no grupo seminobre. Esse menor desenvolvimento vegetativo ajuda a explicar a baixa PT e PC obtida nesse estudo, pois quanto menor a área foliar, menor é a interceptação de radiação e, consequentemente, dos níveis de fotossíntese líquida, resultando em menor acúmulo de massa seca pelas plantas (Larcher, 2000; Taiz \& Zeiger, 2008).

O maior potencial produtivo da cultivar São Valentim está relacionado ao maior número de bulbilhos por bulbo, pois o tamanho do bulbilho, estimado pelo comprimento e largura do mesmo, não diferiu das demais cultivares (Tabela 3). As cultivares Chinês Real e Hozan, que apresentaram valores semelhantes à cultivar São Valentim para número, comprimento e largura do bulbilho, apresentaram PT e PC inferiores à cultivar São Valentim, podendo-se atribuir esse fato à maior sensibilidade ao superbrotamento e redução da qualidade dos bulbos. Entretanto, o aumento do número de bulbilhos não garante maior valor comercial da produção, sendo apenas um indicativo, pois dependendo da quantidade de bulbilhos por bulbo, ocorre a depreciação comercial devido à presença de bulbilhos "palito" (Resende, 1993).

As baixas produtividades e o elevado índice de superbrotamento indicam a necessidade de mais pesquisas para avaliação da adaptabilidade das cultivares de alho disponíveis para o plantio, devendo-se variar a época de cultivo e o manejo, como adubação e vernalização. Nas condições de cultivo do presente estudo, a cultivar São Valentim demonstrou melhor adaptação às condições climáticas da região, demonstrando elevado potencial para cultivo na região.

Conclui-se que: i) não há diferença na produção comercial de bulbos entre as cultivares do grupo nobre e seminobre na região; ii) a cultivar São Valentim é a mais produtiva e demonstra elevado potencial de cultivo na região; iii) as cultivares do grupo nobre produzem bulbos de melhor qualidade do que as cultivares do grupo seminobre, com destaque para as cultivares Chonan e Ito; e iv) o aumento do número de bulbilhos por bulbo e da largura da folha e do pseudocaule são indicativos de aumento de produtividade.

\section{REFERÊNCIAS}

BACKES C; LIMA CPD; GODOY LJGD; VILLAS BÔAS RL; IMAIZUMI I. 2008. Coloração verde nas folhas da cultura do alho vernalizado em resposta à adubação nitrogenada. Bragantia 67: 491-498.

BUSO GSC; PAIVA MR; TORRES AC; RESENDE FV; FERREIRA MA; BUSO ANJ. 2008. Genetic diversity studies of Brazilian garlic cultivars and quality control of garlic clover production. Genetics and Molecular Research 7: 534-541.

CARMO CAS. 1984. Efeitos da cobertura do solo e da frequência de irrigação na cultura do alho (Allium sativum L.). em dois locais de altitudes diferentes do Estado do Espírito Santo. Viçosa: UFV. 61p (Tese doutorado).

CONAB. Companhia Nacional de Abastecimento. Alho. Disponível em: http://www.conab.gov.br/OlalaCMS/ uploads/arquivos/11_03_11_17_12_15 alhojaneiro2011. pdf. Acessado em 20 de janeiro de 2012.

EMBRAPA. 2006. Sistema brasileiro de classificação de solos. Rio de Janeiro: Embrapa Solos. 306p.

FEITOSA HDO; JUNQUEIRA RM; GUERRA JGM; TEIXEIRA MG; RESENDE FV. 2009. Avaliação de cultivares de alho em três regiões do Estado do Rio de Janeiro cultivados sob sistema orgânico. Revista Brasileira de Ciências Agrárias 4: 399-404.

FILGUEIRA FAR. 2008. Novo manual de olericultura. Viçosa: UFV. 421p.

LARCHER W. 2000. Ecofisiologia Vegetal. São Carlos: RiMa. 529p.

MACÊDO FS; SOUZA RJD; PEREIRA GM. 2006. Controle de superbrotamento e produtividade de alho vernalizado sob estresse hídrico. Pesquisa Agropecuária Brasileira 41: 629-635.

MENEZES SOBRINHO JA. 1997. Cultivo do alho (Allium sativum). Brasília: EMBRAPA/ CNPH. 23p.

MOTA JH; SOUZA RJD; YURI JE; REZENDE GMD; TEIXEIRA IR. 2005. Similaridade morfológica de cultivares de alho (Allium sativum L.). Revista Cientifica Eletrônica de Agronomia 4:8.

MOTA JH; YURI JE; RESENDE GM; SOUZA RJD. 2006. Similaridade genética de cultivares de alho pela comparação de caracteres morfológicos, físico-químicos, produtivos e moleculares. Horticultura Brasileira 24: 156-160.

MOTA JHN; SOUZA RJD; YURI JE; RESENDE GMD; PAIVA LV. 2004. Diversidade genética de cultivares de alho (Allium sativum L.) por meio de marcador molecular RAPD. Ciência e Agrotecnologia 28: 764-770.

RESENDE GM. 1993. Comportamento de cultivares de alho (Allium sativum L.) no Norte de Minas Gerais. Horticultura Brasileira 11: 93.

RESENDE GM; SOUZA RJ. 2001. Efeitos de tipos de bulbos e adubação nitrogenada sobre a produtividade e características comerciais do alho cv. "Quitéria". Horticultura Brasileira 19: $320-323$.

RESENDE GMD; CHAGAS SJLDR; PEREIRA LV. 2003. Características produtivas e qualitativas de cultivares de alho. Horticultura Brasileira 21: 686-689.

RESENDE JTV; MORALES RGF; RESENDE FV; FARIA MV; SOUZA RJ; MARCHESE A. 2011. Garlic vernalization and planting dates in Guarapuava. Horticultura Brasileira 29: 193-198.

SOUZA RJD; MACÊDO FS. 2004. Vernalização de cultivares de alho nobre na região de Lavras. Horticultura Brasileira 22: 651-654.

SOUZA RJD; MACÊDO FS. 2009. Cultura do alho: técnicas modernas de produção. Lavras: UFLA. $181 \mathrm{p}$.

TAIZ L; ZEIGER E. 2008. Fisiologia vegetal. Porto Alegre: Artmed. 819p.

TRANI PE; FOLTRAN DIE; CAMARGO MNSD; TIVELLI SOW; PASSOS FA. 2008. Produtividade de cultivares de alho na região paulista de Tietê. Bragantia 67: 713-716.

TREVISAN JN; MARTINS GAK; SANTOS NRZ. 1997. Influência da época de plantio na produção de classes de bulbos comerciais de cultivares de alho (Allium sativum L.) em Santa Maria, RS. Ciência Rural 27: 7-11. 